\title{
MULTIBASELINE GRADIENT AMBIGUITY RESOLUTION TO SUPPORT MINIMUM COST FLOW PHASE UNWRAPPING
}

\author{
Marie Lachaise (1), Richard Bamler (1)(2), Fernando Rodriguez Gonzalez (2)
}

\author{
(1) German Aerospace Center (DLR), Remote Sensing Technology Institute, 82234 Weßling, Germany \\ (2) Technische Univesität München, Remote Sensing Technology, Arcisstr. 21, 80333 Munich, Germany
}

\begin{abstract}
The TanDEM-X Mission has as primary objective to generate a high resolution global Digital Elevation Model. This paper proposes a new method for multibaseline Phase Unwrapping which is the critical point of this generation. We propose to combine both Minimum Cost Flow (MCF) and Maximum a Posteriori (MAP) estimation. The latter is used to solve phase gradient ambiguities. The problem is posed as an energy minimization one and solved using Belief Propagation (BP) which is an iterative process. Nevertheless, although very good results are obtained on loopy graphs, it is not guaranteed to converge. Thus, phase unwrapping of the most accurate interferogram is finally performed with the MCF algorithm and takes as input the unwrapped gradients.
\end{abstract}

Index Terms - TanDEM-X, multibaseline phase unwrapping, Minimum Cost Flow, Belief Propagation

\section{INTRODUCTION}

The TanDEM-X Mission started in June 2010. Its primary objective is the generation of a consistent global Digital Elevation Model (DEM) with an unprecedented accuracy. The whole land mass will be mapped with two different baselines in order to reduce the difficulty of phase unwrapping while achieving the required accuracy. Phase unwrapping is a crucial step to obtain this high quality DEM.

Classically DEMs have been generated from a single interferogram. Constantini [1] proposed a branch cut based phase unwrapping algorithm (Minimum Cost Flow, MCF). However, problems could arise in this process. One of the novelties of the TanDEM-X Mission is its dual baseline approach. Several methods for multibaseline phase unwrapping based on Maximum Likelihood Estimation (MLE) have been developed like in [2]. Most of them work on a pixel-by-pixel basis, not accounting for overall structural information. Reconstruction quality could be increased using and a prior [3]. Nonetheless computation is heavily increased. Moreover, in our case where only two interferograms are available during the first two mission years, this method is still not robust enough. Frey [4] proposed a new phase unwrapping method (for a single interferogram) using loopy Belief Propagation (BP, [5]) to solve the MAP problem. Although BP is guaranteed to converge only in trees, he showed it can produce excellent results in very loopy graphs.

The method we propose here combines both MCF and MAP. Firstly, MAP is used to unwrap phase gradients because the required search interval is much smaller than for the phase. Once the problem of MAP is rewritten as an energy minimization one, we can apply BP to solve it. However, due its possible non-convergence on loopy graphs, we couple it to the MCF. It unwraps the most accurate interferogram, i.e. the one with the larger baseline. Since gradient MAP has already solved or reduced the ambiguity error in gradient estimates, phase unwrapping becomes quite trivial. As a consequence, the advantages of both MCF and MAP are efficiently combined into a single robust framework.

\section{MULTIBASELINE GRADIENT AMBIGUITY RESOLUTION TO SUPPORT MCF ALGORITHM}

The objective of phase unwrapping is to derive an estimate $\hat{\phi}(i, k)$ of the true phase $\phi(i, k)$ given its wrapped values $\psi(i, k)=W\{\phi(i, k)\} \in[-\pi, \pi)$. Our objective is to enhance gradient estimates $\hat{\nabla} \psi(i, k)$ of $\nabla \phi(i, k)$ using MAP. If necessary, these estimates are corrected and then integrated to determine $\hat{\phi}(i, k)$ with the help of the MCF algorithm.

\subsection{Unwrapped gradient estimation}

\subsubsection{Multibaseline gradient likelihood}

The multibaseline gradient likelihood consists of combining the gradient estimates of two or more interferograms $\left\{\psi_{l}\right\}_{l \in\{1, \ldots, L\}}$ in order to reduce the gradient ambiguity. Gradients $\hat{\nabla} \psi(i, k)$ are estimated by computing partial derivatives of $\psi(i, k)$ which are wrapped back if they exceed 
$\pm \pi$. Their probability density function $p d f\left(\delta_{\phi}\right)$ (in $i$ direction) is [6]

$$
p d f\left(\delta_{\phi_{i}}\right)[i, k]=p d f(\phi ; \gamma, L)[i+1, k] * p d f(-\phi ; \gamma, L)[i, k](1)
$$

where $p d f(\phi ; \gamma, L)$ is the $p d f$ of an interferogram sample given by (2) and * stands for convolution.

$$
\begin{aligned}
p d f(\widehat{\phi} ; \gamma, L)= & \frac{\Gamma(L+1 / 2)\left(1-|\gamma|^{2}\right)^{L}|\gamma| \cos \left(\phi-\phi_{0}\right)}{2 \sqrt{\pi} \Gamma(L)\left(1-|\gamma|^{2} \cos ^{2}\left(\phi-\phi_{0}\right)\right)^{L+1 / 2}}+ \\
& \frac{\left(1-|\gamma|^{2}\right)^{L}}{2 \pi}{ }_{2} F_{1}\left(L, 1 ; 0.5 ;|\gamma|^{2} \cos ^{2}\left(\phi-\phi_{0}\right)\right)
\end{aligned}
$$

Once one of the interferograms has been selected as reference $(c=1)$, the gradient distributions of the other interferograms may be scaled by the baseline ratios $a_{c}=B_{1} / B_{c}$, where $B_{c}$ is the baseline of the interferogram $c$. The multibaseline gradient likelihood function or joint $p d f$ of the gradient is (in $i$-direction) then

$$
p d f\left(\hat{\delta}_{\phi_{i}}\right)[i, k]=\prod_{c}^{c} p d f\left(\phi_{c} ; \gamma_{c}, L\right)[i+1, k] * p d f\left(-\phi_{c} ; \gamma_{c}, L\right)[i, k]
$$

where $C$ is the number of interferograms.

Since it is very unlikely to have a very high gradient, the considered interval is then reduced to three or five cycles of the reference interferogram i.e. the one with the smallest baseline. As a result, the likelihood calculation is much faster than on the phase.

Nevertheless, although the maximum of this likelihood (Maximum Likelihood Estimate) eliminates or at least reduces the ambiguity problems due to the wrapping operator, it amplifies the noise contribution. Fig. 1 shows how much the joint pdf can change regarding the coherence and the shifts between every gradients of a pixel. Thus, depending on the difference of the acquired gradients, the number of significant peaks of the joint distribution may considerably vary (fig.2) and therefore leads to a wrong estimate.

\subsubsection{Prior and energy minimization}

Another point to be taken in consideration is the link between gradients in both directions. In this sense, the conservative condition of a gradient field helps us and leads to the affirmation: we want to find the unwrapped gradients with the constraint that the sum of the gradients around every loop must be zero which is called the zero curl constraint and is written

$$
\left(\delta_{\phi_{i}}[i, k]+\delta_{\phi_{k}}[i+1, k]-\delta_{\phi_{i}}[i, k+1]-\delta_{\phi_{k}}[i, k]\right)=0(4) .
$$

We introduce then a prior modeled by a Markov Random Field $\Omega\left(\mathrm{x}_{\mathrm{i}}, \mathrm{x}_{\mathrm{j}}\right)$ which introduces a compatibility between neighboring variables. According to Bayes' rule, the posterior $P(\boldsymbol{x}, \boldsymbol{y})$ can be written as the product of a likelihood $\Lambda\left(\mathrm{x}_{\mathrm{i}}, \mathrm{y}_{\mathrm{i}}\right)$ and a prior $\Omega\left(\mathrm{x}_{\mathrm{i}}, \mathrm{x}_{\mathrm{j}}\right)$ i.e.

$$
P(\mathbf{x}, \mathbf{y})=\prod_{p} \Lambda\left(x_{p}, y_{p}\right) \prod_{(i, k)} \Omega\left(x_{i}, x_{k}\right)(5)
$$
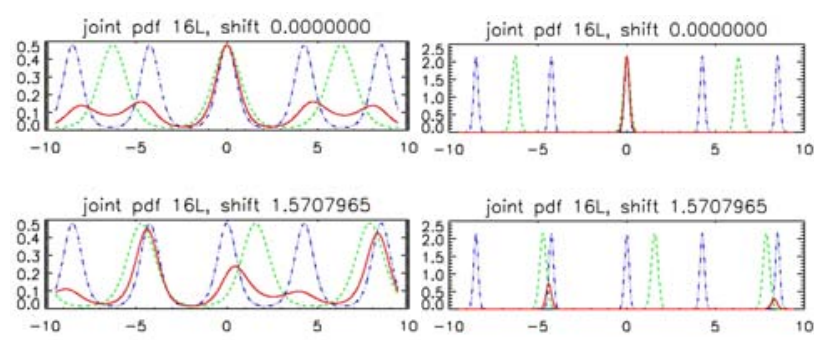

Fig. 1. Example of $p d f$ (dashed) and joint $p d f$ (solid) for two different configurations of the gradients $p d f$ for $\gamma=0.3$ and $\gamma=0: 8$
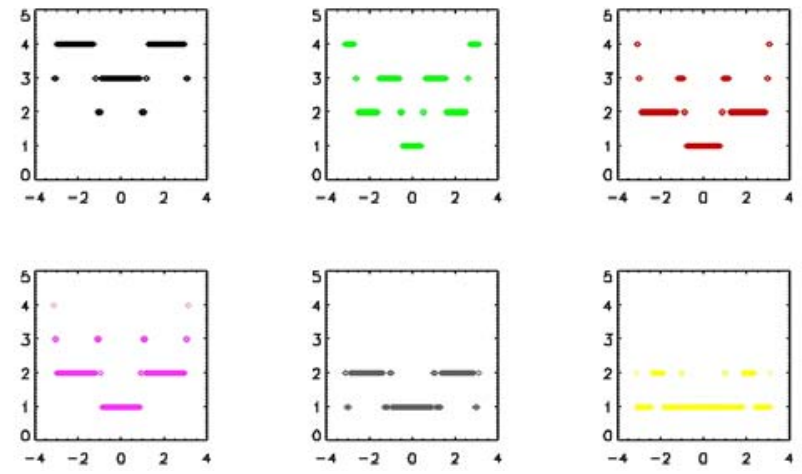

Fig. 2. Expected numbers of significant peaks according to the difference between both gradients for different $\gamma$ (from left to right, first line: 0.2, 0.4, 0.6; second line: 0.7, 0.8, and 0.9).

For our problem, in (5) the likelihood is then the product of the joint $p d f$ of both directions and the prior is represented by a probability model on the gradients that satisfies the zero curl constraint. The posterior probability can be written as

$$
P\left(\delta_{\phi_{i}}, \delta_{\phi_{k}}\right)=\prod_{i, k}^{P} P\left(\delta_{\phi_{i}}\right)[i, k] \prod_{i, k}^{P} P\left(\delta_{\phi_{k}}\right)[i, k]
$$

$\prod_{i, k}^{P} \prod_{c}^{C} P\left(\delta_{\phi_{i}, c}[i, k]+\delta_{\phi_{k}, c}[i+1, k]-\delta_{\phi_{i}, c}[i, k+1]-\delta_{\phi_{k}, c}[i, k]\right)$

with $P\left(\delta_{\phi_{i}}\right)[i, k]$ and $P\left(\delta_{\phi_{k}}\right)[i, k]$ follow (3) and

$$
\begin{aligned}
P\left(\delta_{\phi_{i}, c}[i, k]+\delta_{\phi_{k}, c}[i+1, k]-\delta_{\phi_{i}, c}[i, k+1]-\delta_{\phi_{k}, c}[i, k]\right) \\
=\left\{\begin{array}{c}
1 \text { if }(4) \text { ture } \\
0 \text { if not }
\end{array}\right.
\end{aligned}
$$

Taking the negative logarithm of (5), we obtain

$$
E(\mathbf{x}, \mathbf{y})=\sum_{p}-\log \Lambda\left(x_{p}, y_{p}\right)+\sum_{(i, k)}-\log \Omega\left(x_{i}, x_{k}\right) \text { (8) }
$$

To find the most likely solution of (5), we need to minimize the energy 


$$
E(\mathbf{x}, \mathbf{y})=E_{d}+E_{s}=\sum_{p} D\left(x_{p}, y_{p}\right)+\sum_{(i, k)} V\left(x_{i}, x_{k}\right) \text { (9). }
$$

The first term $E_{d}$ is the data energy or data penalty. The second term is $E_{s}$ is the prior energy or discontinuity cost.

\subsubsection{Belief Propagation}

The joint distribution (6) can be described by a graphical model shown in fig 3. Red (dark) points are the partial derivatives over range and azimuth between two purple (light) points which are the phase measurements. Black points are the zero curl constraint and are connected to their respective four gradients that they constraint to sum to 0 .

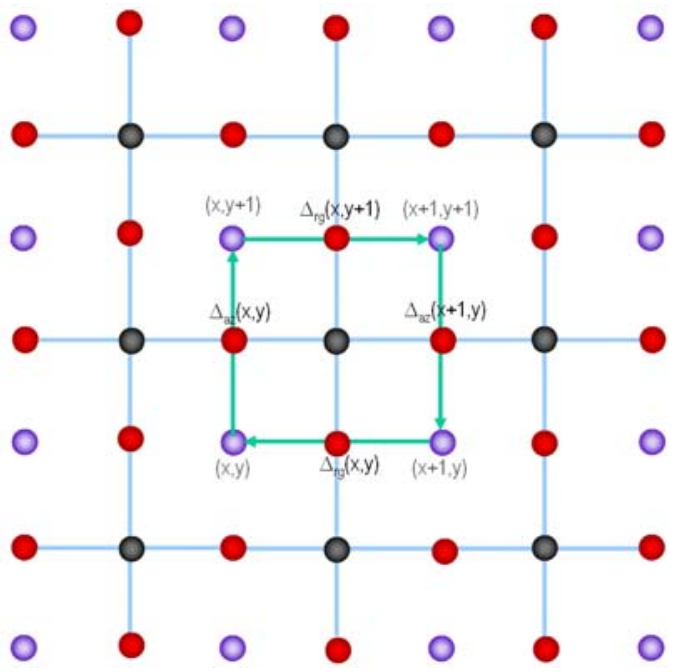

Fig. 3. Graphical model for the zero curl constraint

Belief propagation computes messages which are passed both directions on every edge in the network. The elements of the vectors correspond to the different log likelihood values. There are two types of messages: the constraint to gradient message (fig. 4a) and the gradient to constraint message (fig. $4 b$ ).

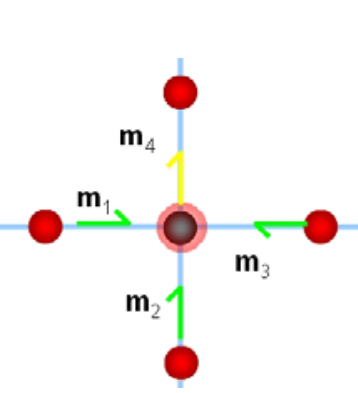

(a)

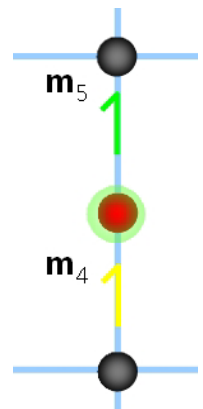

(b)

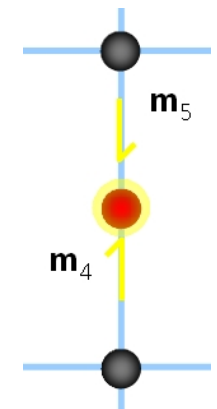

(c)
Fig. 4. Message passing: (a) constraint to gradient message (b) gradient to constraint message (c) resulting message

Constraint to gradient messages are calculated following this equation

$$
\begin{gathered}
m_{4 i}=\min \left(\sum_{j} \sum_{k} \sum_{l}\left(m_{1 j}+m_{2 k}+m_{3 l}\right)+\right. \\
\left.E\left(\delta_{\phi_{k}}+\delta_{\phi_{i+1}}-\delta_{\phi_{k+1}}-\delta_{\phi_{i}}\right)\right)
\end{gathered}
$$

where $E\left(\delta_{\phi_{k}}+\delta_{\phi_{i+1}}-\delta_{\phi_{k+1}}-\delta_{\phi_{i}}\right)$ is 0 if the sum of the gradients is 0 and $+\infty$ if not.

Gradient to constraint message are simply

$$
m_{5 i}=-\log p d f_{i}+m_{4 i}(11)
$$

Finally, the final belief of a gradient is the sum of all incoming messages in that gradient node (fig. 4c) and the minimum gives the estimate of the unwrapped gradient.

The message updating schedule is a "up-down-left-right" [7].

\subsection{Minimum Cost Flow algorithm supported by unwrapped gradients}

The multibaseline gradient estimates do not exactly correspond to any of the ambiguities of the original interferograms. In order to allow the application of the MCF in the second stage of the algorithm, gradient estimates are rounded to their nearest ambiguities.

At DLR, a new MCF implementation optimized both in terms of memory and time consumption had been developed [8]. Its efficiency has been proved during the SRTM mission. This algorithm follows a global approach incorporating the prior that the gradient of the unwrapped phase should be a conservative field (but does not take any probability into account). It is based on gradient estimates, whose ambiguities are corrected according to a given cost function. The MCF approach solves the following global minimization problem

$$
\min _{d_{i}, d_{k}}\left\{\sum_{i, k} c_{i}(i, k)\left|d_{i}(i, k)\right|+\sum_{i, k} c_{k}(i, k)\left|d_{k}(i, k)\right|\right\}
$$

In the usual MCF approach, $d_{i}(i ; k)$ and $d_{k}(i ; k)$ are the residue fields and have values equal to $0 ;+2 \pi ;-2 \pi$. They are used to correct the gradient estimate, making it conservative. In our approach, new residue fields are calculated with the help of the unwrapped gradients. Since the interval of possible gradient estimates has been extended, their values can be now integer multiples of $2 \pi$. Finally, adapted cost functions $c_{i}(i ; k)$ and $c_{k}(i ; k)$ have been developed.

\section{RESULTS}

Multibaseline interferograms have been simulated using a DEM obtained from a repeat-pass TerraSAR-X (TSX) interferogram and real TSX geometrical parameters. Hence our data is realistic regarding geometrical aspects, but 
without any atmospheric artifacts. Moreover, the level of noise has been controlled. We simulated interferograms with two different baselines. The first interferogram has a height of ambiguity of $40.1 \mathrm{~m} /$ cycle and the second one of $27.0 \mathrm{~m} /$ cycle, analogous to TanDEM-X operational configuration. Search interval for gradient MAP is three cycles of the interferogram which is taken as reference. Multibaseline gradient estimation has been performed in order to remove the gradient ambiguity for each interferogram (see fig. 4a). The unwrapped gradients in range and azimuth are used as inputs to MCF. New residue fields are obtained (fig. 4c and 4d). It can be observed that most of the long branch-cuts are successfully removed. Hence the resulting unwrapped phase exhibits less errors. Concretely, in fig. 4c, there is a long and obviously erroneous branch cut in the lower part. It has been efficiently corrected by our approach (fig. 4d).

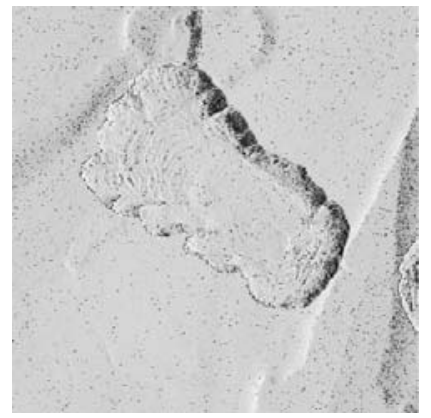

(a)

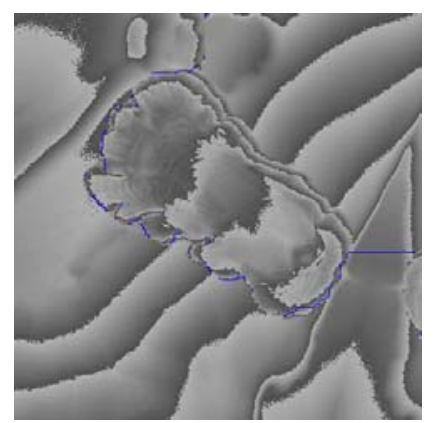

(c)

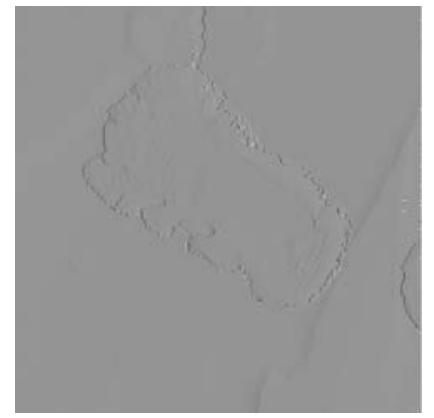

(b)

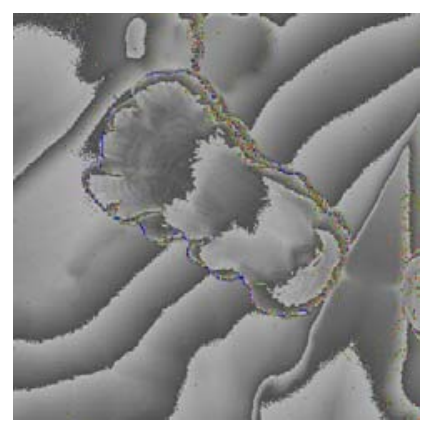

(d)
Fig. 5. Results obtained with simulated data from a TSX DEM $(\gamma=0: 8)$ : (a) dual-baseline unwrapped gradient in range, (b) derived costs obtained from the pdf distributions, (c) residues and branchcuts obtained from the MCF algorithm without unwrapped gradient

\section{REFERENCES}

[1] M. Constantini: A novel phase unwrapping method based on network programming, IEEE Transactions on Geoscience and Remote Sensing. Vol. 36, Issue 3, pp. 813 - 821, May 1998

[2] M. Eineder and N. Adam, "A maximum-likelihood estimator to simultaneously unwrap, geocode, and fuse sar interferograms from different viewing geometries into one digital elevation model," Transactions on Geoscience and Remote Sensing, vol. 43, pp. 24 36, January 2005.

[3] G. Ferraiuolo, V. Pascazio, and G. Schirinzi, "Maximum a posteriori estimation of height profiles in insar imaging," Geoscience and Remote Sensing Letters, vol. 1, no. 2, pp. 66-70, April 2004.

[4] B. J. Frey, R. Koetter and N. Petrovic, "Very Loopy Belief Propagation for Unwrapping Phase Images", Neural Information Processing Systems 14, 2001

[5] J. Yedidia, W.T. Freeman, and Y. Weiss, "Understanding Belief Propagation and its Generalizations", International Joint Conference on Artificial Intelligence (IJCAI 2001), 2001

[6] R. Bamler, N. Adam, G.W. Davidson, and D. Just, "Noiseinduced slope distortion in 2-d phase unwrapping by linear estimators with application to sar interferometry," Transactions on Geoscience and Remote Sensing, vol. 36, pp. 913-921, May 1998.

[7] M.F. Tappen, W.T. Freeman, "Comparison of Graph Cuts with Belief Propagation for Stereo, using Identical MRF Parameters," ICCV, vol. 2, pp.900, Ninth IEEE International Conference on Computer Vision (ICCV'03) - Volume 2, 2003

[8] M. Eineder, M. Hubig, and B. Milcke, "Unwrapping large interferograms using the minimum cost flow algorithm," in Geoscience and Remote Sensing Symposium Proceedings. IEEE, 1998, pp. 83-87. 